Supporting Information for:

\title{
Molecular Origin and Electrochemical Influence of Capacitive Surface States on Iron Oxide Photoanodes
}

\author{
Yelin $\mathrm{Hu}^{\mathrm{a}, \mathrm{b}}$, Florent Boudoire ${ }^{\mathrm{a}, \mathrm{c}}$, Iris Hermann-Geppert ${ }^{\mathrm{d}, \mathrm{e}}$, Peter Bogdanoff ${ }^{\mathrm{f}}$, George \\ Tsekouras $^{\mathrm{a}}$, Bongjin Simon Mun ${ }^{\mathrm{g}}$, \\ Giuseppino Fortunato $^{\mathrm{h}}$, Michael Graetzel $^{\mathrm{b}}$, Artur Braun $^{\mathrm{a}}$
}

${ }^{1}$ Laboratory for High Performance Ceramics, Empa, Swiss Federal Laboratories for Materials Science and Technology, CH-8600 Dübendorf, Switzerland

${ }^{2}$ Laboratory of Photonics and Interfaces, Institute of Chemical Sciences and Engineering, Swiss Federal Institute of Technology, CH-1015 Lausanne, Switzerland

${ }^{3}$ Department of Chemistry, University of Basel, Spitalstr. 51, CH-4056 Basel, Switzerland

${ }^{4}$ Institute for Materials Research, Sustainable Energy Technology, Helmholtz-Zentrum Geesthacht, D-21502 Geesthacht, Germany

${ }^{5}$ Institute for Materials Technology, Helmut-Schmidt University, D-22043 Hamburg, Germany

${ }^{6}$ Institute for Solar Fuels, Helmholtz-Zentrum Berlin für Materialien und Energie, D-14109 Berlin, Germany

${ }^{7}$ Department of Physics and Photon Science, Ertl Center for Electrochemistry and Catalysis, Gwangju Institute of Science and Technology, Gwangju, Korea

${ }^{8}$ Protection and Physiology, Empa, Swiss Federal Laboratories for Materials Science and Technology, CH-9014 St. Gallen, Switzerland

*Corresponding author: artur.braun@alumni.ethz.ch 


\section{Molecular Origin and Electrochemical Influence of capacitive surface}

states on iron oxide photoanodes
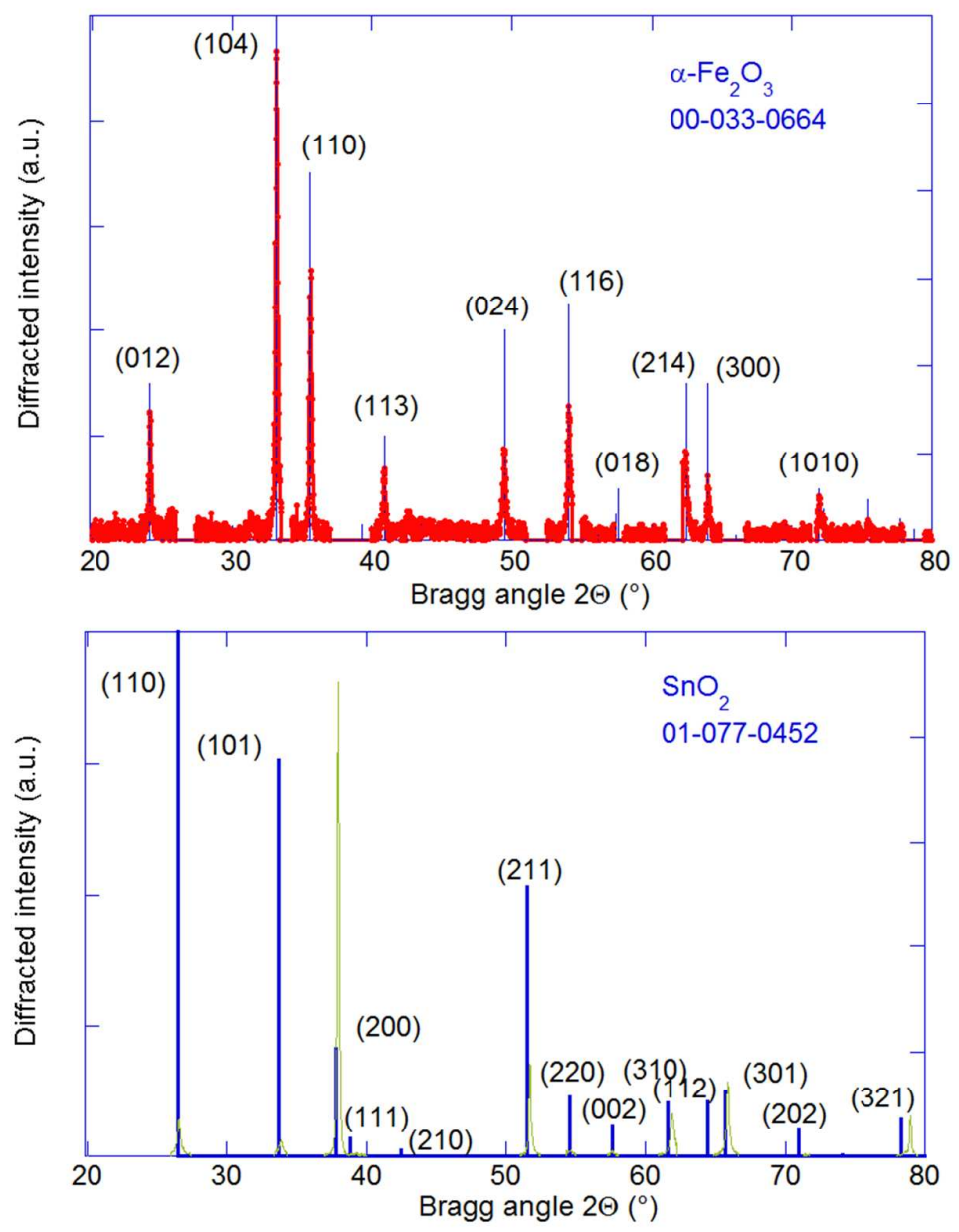

Figure S1. X-ray diffractogram $(\lambda=1.54056 \AA)$ of iron oxide film deposited on FTO glass substrate. Top panel shows iron oxide Bragg reflections and peak positions of JCPDS 00-033-0664 reference. Bottom panel shows Bragg reflections from the FTO substrate and peak positions of JCPDS 01-077-0452 of $\mathrm{SnO}_{2}$ reference. 


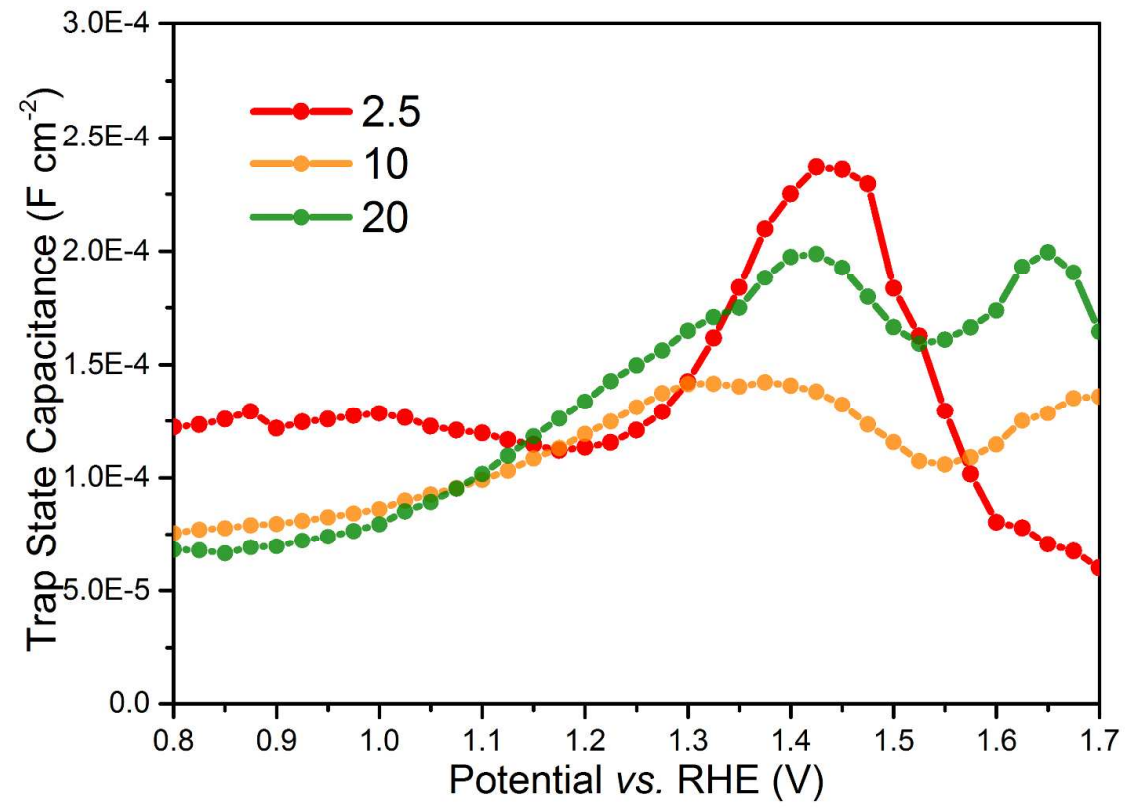

Figure S2. Trapped surface state capacitance of hematite electrode vs. applied potential in $1 \mathrm{M} \mathrm{KOH}$ under 1 Sun illumination with oxygen plasma treatment times (min).

Table S1. Fit parameters for EIS data of pristine and plasma treated hematite samples under selected potential

\begin{tabular}{|c|c|c|c|c|c|c|c|}
\hline \multicolumn{8}{|l|}{0 min } \\
\hline $\begin{array}{l}\text { Potential } \\
\text { (V) }\end{array}$ & $\mathrm{R}_{\mathrm{S}}(\Omega)$ & $\mathrm{R}_{\text {trapping }}(\Omega)$ & $\mathrm{R}_{\mathrm{ct} \text { trap }}(\Omega)$ & $\begin{array}{l}\mathrm{CPE}_{\text {trap }}: \mathrm{T} \\
\left(\mathrm{F} \mathrm{cm}^{-2} \mathrm{~s}^{\mathrm{P}-1}\right)\end{array}$ & $\mathrm{CPE}_{\text {trap }}: \mathrm{P}(-)$ & $\begin{array}{l}\mathrm{CPE}_{\text {bulk }}: \mathrm{T} \\
\left(\mathrm{F} \mathrm{cm}^{-2} \mathrm{~s}^{\mathrm{P}-1}\right)\end{array}$ & $\mathrm{CPE}_{\text {bulk }}: \mathrm{P}(-)$ \\
\hline 1.7 & 19.76 & 16.36 & 46.81 & 0.000163 & 0.8 & $5.47 \mathrm{E}-05$ & 0.8 \\
\hline 1.675 & 17.58 & 21.29 & 81.1 & 0.000208 & 0.8 & $7.95 \mathrm{E}-05$ & 0.8 \\
\hline 1.65 & 17.97 & 35.94 & 207 & 0.000184 & 0.8 & $7.99 \mathrm{E}-05$ & 0.8 \\
\hline 1.625 & 18.3 & 64.92 & 534.9 & 0.000157 & 0.8 & 7.72E-05 & 0.8 \\
\hline
\end{tabular}




\begin{tabular}{|c|c|c|c|c|c|c|c|}
\hline 1.6 & 18.41 & 117.6 & 1176 & 0.000125 & 0.8 & $6.86 \mathrm{E}-05$ & 0.8 \\
\hline 1.575 & 18.3 & 218.4 & 1862 & $9.67 \mathrm{E}-05$ & 0.8 & $5.95 \mathrm{E}-05$ & 0.8 \\
\hline 1.55 & 17.97 & 413.9 & 2124 & $7.16 \mathrm{E}-05$ & 0.8 & $5.18 \mathrm{E}-05$ & 0.8 \\
\hline 1.525 & 17.57 & 851.9 & 1842 & $5.31 \mathrm{E}-05$ & 0.8 & $4.61 \mathrm{E}-05$ & 0.8 \\
\hline 1.5 & 17.42 & 2283 & 500.9 & 0.000761 & 0.8 & $4.31 \mathrm{E}-05$ & 0.8 \\
\hline 1.475 & 17.6 & 2395 & 294.3 & 0.001926 & 0.8 & $3.47 \mathrm{E}-05$ & 0.81999 \\
\hline 1.45 & 17.95 & 2219 & 326.3 & 0.001506 & 0.8 & $2.96 \mathrm{E}-05$ & 0.83655 \\
\hline 1.425 & 18.03 & 2156 & 260.9 & 0.003742 & 1 & $2.86 \mathrm{E}-05$ & 0.83829 \\
\hline 1.4 & 18.2 & 1954 & 390.5 & 0.003848 & 0.8 & $2.83 \mathrm{E}-05$ & 0.83877 \\
\hline 1.375 & 18.22 & 1737 & 342.2 & 0.002842 & 0.8 & $2.83 \mathrm{E}-05$ & 0.83799 \\
\hline 1.35 & 18.4 & 1557 & 357.9 & 0.002791 & 0.8 & $2.92 \mathrm{E}-05$ & 0.83358 \\
\hline 1.325 & 18.58 & 1376 & 360.5 & 0.002276 & 0.8 & $2.97 \mathrm{E}-05$ & 0.8315 \\
\hline 1.3 & 18.72 & 1238 & 391.3 & 0.002172 & 0.8 & $3.04 \mathrm{E}-05$ & 0.82893 \\
\hline 1.275 & 18.79 & 1090 & 397.9 & 0.001748 & 0.8 & $3.07 \mathrm{E}-05$ & 0.82796 \\
\hline 1.25 & 18.83 & 970.8 & 414.2 & 0.001492 & 0.8 & $3.13 \mathrm{E}-05$ & 0.82635 \\
\hline 1.225 & 18.89 & 873 & 418.7 & 0.001165 & 0.8 & $3.26 \mathrm{E}-05$ & 0.82266 \\
\hline 1.2 & 18.86 & 801 & 418.9 & 0.001009 & 0.8 & $3.47 \mathrm{E}-05$ & 0.8164 \\
\hline 1.175 & 16.38 & 661.5 & 373 & 0.001004 & 0.8 & $4.31 \mathrm{E}-05$ & 0.82812 \\
\hline 1.15 & 16.36 & 605.8 & 389.8 & 0.000739 & 0.8 & $4.69 \mathrm{E}-05$ & 0.82065 \\
\hline 1.125 & 16.27 & 577.4 & 422.3 & 0.000603 & 0.8 & $5.31 \mathrm{E}-05$ & 0.80907 \\
\hline 1.1 & 16.28 & 545.3 & 489 & 0.000444 & 0.8 & $5.76 \mathrm{E}-05$ & 0.80294 \\
\hline 1.075 & 16.33 & 512.8 & 600.6 & 0.00033 & 0.8 & $6.11 \mathrm{E}-05$ & 0.8 \\
\hline
\end{tabular}




\begin{tabular}{|c|c|c|c|c|c|c|c|}
\hline 1.05 & 16.44 & 488.3 & 744.8 & 0.000255 & 0.8 & $6.38 \mathrm{E}-05$ & 0.8 \\
\hline 1.025 & 16.53 & 466.3 & 971.6 & 0.000202 & 0.8 & $6.68 \mathrm{E}-05$ & 0.8 \\
\hline 1 & 16.61 & 439.9 & 1311 & 0.000159 & 0.8 & 7E-05 & 0.8 \\
\hline 0.975 & 16.67 & 423.1 & 1771 & 0.000133 & 0.8 & 7.39E-05 & 0.8 \\
\hline 0.95 & 16.79 & 405.8 & 2443 & 0.000113 & 0.8 & $7.8 \mathrm{E}-05$ & 0.8 \\
\hline 0.925 & 16.87 & 389.8 & 3399 & $9.77 \mathrm{E}-05$ & 0.8 & 8.29E-05 & 0.8 \\
\hline 0.9 & 16.94 & 380.2 & 4759 & $8.57 \mathrm{E}-05$ & 0.8 & $8.87 \mathrm{E}-05$ & 0.8 \\
\hline 0.875 & 17.01 & 364.9 & 6828 & 7.62E-05 & 0.8 & $9.52 \mathrm{E}-05$ & 0.8 \\
\hline 0.85 & 17.04 & 354.3 & 9503 & $6.86 \mathrm{E}-05$ & 0.8 & 0.000103 & 0.8 \\
\hline 0.825 & 17.09 & 338.7 & 12581 & $6.22 \mathrm{E}-05$ & 0.8 & 0.000112 & 0.8 \\
\hline 0.8 & 17.1 & 317.6 & 16774 & $5.79 \mathrm{E}-05$ & 0.8 & 0.000123 & 0.8 \\
\hline \multicolumn{8}{|l|}{$2.5 \mathrm{~min}$} \\
\hline $\begin{array}{l}\text { Potential } \\
\text { (V) }\end{array}$ & $\mathrm{R}_{\mathrm{S}}(\Omega)$ & $\mathrm{R}_{\text {trapping }}(\Omega)$ & $\mathrm{R}_{\mathrm{ct}, \text { trap }}(\Omega)$ & $\begin{array}{l}\mathrm{CPE}_{\text {trap }}: \mathrm{T} \\
\left(\mathrm{F} \mathrm{cm}^{-2} \mathrm{~s}^{\mathrm{P}-1}\right)\end{array}$ & $\mathrm{CPE}_{\text {trap }}: \mathrm{P}(-)$ & $\begin{array}{l}\mathrm{CPE}_{\text {bulk }}: \mathrm{T} \\
\left(\mathrm{F} \mathrm{cm}^{-2} \mathrm{~s}^{\mathrm{P}-1}\right)\end{array}$ & $\mathrm{CPE}_{\text {bulk: }}: \mathrm{P}(-)$ \\
\hline 1.7 & 12.35 & 12.5 & 37.1 & 0.000244 & 0.8 & $8.14 \mathrm{E}-05$ & 0.8 \\
\hline 1.675 & 12.9 & 17.86 & 52.05 & 0.000254 & 0.8 & $9.81 \mathrm{E}-05$ & 0.8 \\
\hline 1.65 & 13.41 & 29.69 & 91.63 & 0.000243 & 0.8 & 0.000102 & 0.8 \\
\hline 1.625 & 13.82 & 50.49 & 160 & 0.000241 & 0.8 & 0.000102 & 0.8 \\
\hline 1.6 & 13.99 & 74.61 & 224.5 & 0.000231 & 0.8 & $9.51 \mathrm{E}-05$ & 0.8 \\
\hline 1.575 & 14.25 & 106.8 & 270 & 0.000264 & 0.8 & $8.95 \mathrm{E}-05$ & 0.8 \\
\hline 1.55 & 14.37 & 140.5 & 309.5 & 0.000307 & 0.8 & 8.35E-05 & 0.8 \\
\hline 1.525 & 14.44 & 169.4 & 351.4 & 0.000357 & 0.8 & 7.93E-05 & 0.8 \\
\hline
\end{tabular}




\begin{tabular}{|c|c|c|c|c|c|c|c|}
\hline 1.5 & $1.45 \mathrm{E}+01$ & 184.7 & 394.4 & 0.000386 & 0.8 & $7.65 \mathrm{E}-05$ & 0.8 \\
\hline 1.475 & 14.59 & 194.4 & 433.7 & 0.000456 & 0.8 & $7.50 \mathrm{E}-05$ & 0.8 \\
\hline 1.45 & 14.76 & 197.9 & 502.3 & 0.00046 & 0.8 & $7.45 \mathrm{E}-05$ & 0.8 \\
\hline 1.425 & 14.9 & 200.3 & 575.3 & 0.000458 & 0.8 & $7.42 \mathrm{E}-05$ & 0.8 \\
\hline 1.4 & 14.95 & 199 & 658.7 & 0.000437 & 0.8 & $7.42 \mathrm{E}-05$ & 0.8 \\
\hline 1.375 & 15.07 & 198 & 761.9 & 0.00041 & 0.8 & $7.45 \mathrm{E}-05$ & 0.8 \\
\hline 1.35 & 15.15 & 194.2 & 854 & 0.000369 & 0.8 & 7.47E-05 & 0.8 \\
\hline 1.325 & 15.24 & 192.6 & 953.2 & 0.000332 & 0.8 & $7.56 \mathrm{E}-05$ & 0.8 \\
\hline 1.3 & 15.38 & 191.6 & 1036 & 0.000299 & 0.8 & $7.55 \mathrm{E}-05$ & 0.8 \\
\hline 1.275 & 15.44 & 189.8 & 1070 & 0.000276 & 0.8 & $7.65 \mathrm{E}-05$ & 0.8 \\
\hline 1.25 & 15.53 & 189.4 & 1101 & 0.000262 & 0.8 & $7.75 \mathrm{E}-05$ & 0.8 \\
\hline 1.225 & 15.63 & 189 & 1093 & 0.000253 & 0.8 & $7.91 \mathrm{E}-05$ & 0.8 \\
\hline 1.2 & 15.69 & 187.8 & 1093 & 0.000249 & 0.8 & $8.10 \mathrm{E}-05$ & 0.8 \\
\hline 1.175 & 15.78 & 188.5 & 1083 & 0.000247 & 0.8 & 8.33E-05 & 0.8 \\
\hline 1.15 & 15.86 & 191.3 & 1074 & 0.000251 & 0.8 & $8.61 \mathrm{E}-05$ & 0.8 \\
\hline 1.125 & 15.96 & 193.8 & 1064 & 0.000254 & 0.8 & 8.91E-05 & 0.8 \\
\hline 1.1 & 16.01 & 195.9 & 1059 & 0.000259 & 0.8 & $9.22 \mathrm{E}-05$ & 0.8 \\
\hline 1.075 & 16.01 & 197.3 & 1045 & 0.000261 & 0.8 & $9.57 \mathrm{E}-05$ & 0.8 \\
\hline 1.05 & 16.05 & 198.4 & 1081 & 0.000263 & 0.8 & $9.96 \mathrm{E}-05$ & 0.8 \\
\hline 1.025 & 16.1 & 201.6 & 1163 & 0.000269 & 0.8 & 0.000104 & 0.8 \\
\hline 1 & 16.13 & 202.5 & 1302 & 0.000271 & 0.8 & 0.000109 & 0.8 \\
\hline 0.975 & 16.15 & 202.9 & 1455 & 0.000268 & 0.8 & 0.000115 & 0.8 \\
\hline
\end{tabular}




\begin{tabular}{|c|c|c|c|c|c|c|c|}
\hline 0.95 & 16.14 & 205 & 1633 & 0.000264 & 0.8 & 0.000121 & 0.8 \\
\hline 0.925 & 16.12 & 205.7 & 1880 & 0.000261 & 0.8 & 0.000129 & 0.8 \\
\hline 0.9 & 16.06 & 203.7 & 2167 & 0.000256 & 0.8 & 0.000137 & 0.8 \\
\hline 0.875 & 16.07 & 216.6 & 3215 & 0.000264 & 0.8 & 0.000148 & 0.8 \\
\hline 0.85 & 16.02 & 216.9 & 3816 & 0.000258 & 0.8 & 0.00016 & 0.8 \\
\hline 0.825 & 16.01 & 220.1 & 4573 & 0.000253 & 0.8 & 0.000173 & 0.8 \\
\hline 0.8 & 15.92 & 224.1 & 5231 & 0.00025 & 0.8 & 0.000189 & 0.8 \\
\hline \multicolumn{8}{|l|}{5 min } \\
\hline $\begin{array}{l}\text { Potential } \\
\text { (V) }\end{array}$ & $\mathrm{R}_{\mathrm{S}}(\Omega)$ & $\mathrm{R}_{\text {trapping }}(\Omega)$ & $\mathrm{R}_{\mathrm{ct} \text { trap }}(\Omega)$ & $\begin{array}{l}\mathrm{CPE}_{\text {trap }}: \mathrm{T} \\
\left(\mathrm{F} \mathrm{cm}^{-2} \mathrm{~s}^{\mathrm{P}-1}\right)\end{array}$ & $\mathrm{CPE}_{\text {trap }}: \mathrm{P}(-)$ & $\begin{array}{l}\mathrm{CPE}_{\text {bulk }}: \mathrm{T} \\
\left(\mathrm{F} \mathrm{cm}^{-2} \mathrm{~s}^{\mathrm{P}-1}\right)\end{array}$ & $\mathrm{CPE}_{\text {bulk: }}: \mathrm{P}(-)$ \\
\hline 1.7 & 8.969 & 18.02 & 43.31 & 0.00021 & 0.8 & $9.36 \mathrm{E}-05$ & 0.8 \\
\hline 1.675 & 9.086 & 30.55 & 115.6 & 0.000173 & 0.8 & $9.22 \mathrm{E}-05$ & 0.8 \\
\hline 1.65 & 9.181 & 55.24 & 316.8 & 0.000125 & 0.8 & $9.01 \mathrm{E}-05$ & 0.8 \\
\hline 1.625 & 9.206 & 99.24 & 742.3 & 8.79E-05 & 0.8 & $8.56 \mathrm{E}-05$ & 0.8 \\
\hline 1.6 & 9.166 & 158.6 & 1280 & $5.89 \mathrm{E}-05$ & 0.8 & $8.13 \mathrm{E}-05$ & 0.8 \\
\hline 1.575 & 9.025 & 185.1 & 1647 & $3.71 \mathrm{E}-05$ & 0.8 & 7.67E-05 & 0.8 \\
\hline 1.55 & 8.816 & 121.1 & 1916 & $2.47 \mathrm{E}-05$ & 0.8 & 7.13E-05 & 0.8 \\
\hline 1.525 & 8.631 & 65.74 & 2078 & $2.13 \mathrm{E}-05$ & 0.8 & $6.68 \mathrm{E}-05$ & 0.8 \\
\hline 1.5 & 8.533 & 46.26 & 2137 & $2.1 \mathrm{E}-05$ & 0.8 & $6.45 \mathrm{E}-05$ & 0.8 \\
\hline 1.475 & 8.514 & 39.69 & 2091 & $2.24 \mathrm{E}-05$ & 0.8 & $6.17 \mathrm{E}-05$ & 0.80372 \\
\hline 1.45 & 8.49 & 37.42 & 2043 & $2.33 \mathrm{E}-05$ & 0.8 & $6.21 \mathrm{E}-05$ & 0.80357 \\
\hline 1.425 & 8.468 & 37.16 & 1960 & 2.39E-05 & 0.8 & $6.51 \mathrm{E}-05$ & 0.8 \\
\hline
\end{tabular}




\begin{tabular}{|c|c|c|c|c|c|c|c|}
\hline 1.4 & 8.494 & 44.12 & 1854 & $2.45 \mathrm{E}-05$ & 0.8 & $6.79 \mathrm{E}-05$ & 0.8 \\
\hline 1.375 & 8.511 & 44.82 & 1776 & $2.61 \mathrm{E}-05$ & 0.8 & $6.94 \mathrm{E}-05$ & 0.8 \\
\hline 1.35 & 8.517 & 47.3 & 1672 & $2.79 \mathrm{E}-05$ & 0.8 & 7.17E-05 & 0.8 \\
\hline 1.325 & 8.508 & 46.36 & 1592 & $3 \mathrm{E}-05$ & 0.8 & $7.36 \mathrm{E}-05$ & 0.8 \\
\hline 1.3 & 8.514 & 44.85 & 1536 & $3.18 \mathrm{E}-05$ & 0.8 & $7.55 \mathrm{E}-05$ & 0.8 \\
\hline 1.275 & 8.531 & 49.31 & 1461 & $3.37 \mathrm{E}-05$ & 0.8 & $7.89 \mathrm{E}-05$ & 0.8 \\
\hline 1.25 & 8.599 & 59.79 & 1452 & $3.54 \mathrm{E}-05$ & 0.8 & $8.38 \mathrm{E}-05$ & 0.8 \\
\hline 1.225 & 8.651 & 69.67 & 1467 & $3.8 \mathrm{E}-05$ & 0.8 & $8.85 \mathrm{E}-05$ & 0.8 \\
\hline 1.2 & 8.8 & 106.4 & 1545 & 4.07E-05 & 0.8 & $9.69 \mathrm{E}-05$ & 0.8 \\
\hline 1.175 & 9.021 & 234.8 & 1638 & $4.92 \mathrm{E}-05$ & 0.8 & 0.000109 & 0.8 \\
\hline 1.15 & 9.158 & 367.9 & 1894 & $6.4 \mathrm{E}-05$ & 0.8 & 0.000118 & 0.8 \\
\hline 1.125 & 9.174 & 365.4 & 2351 & $6.57 \mathrm{E}-05$ & 0.8 & 0.000124 & 0.8 \\
\hline 1.1 & 9.077 & 243.8 & 2872 & $6.01 \mathrm{E}-05$ & 0.8 & 0.000126 & 0.8 \\
\hline 1.075 & 9.01 & 180.3 & 3667 & $5.99 \mathrm{E}-05$ & 0.8 & 0.00013 & 0.8 \\
\hline 1.05 & 8.89 & 110.8 & 4578 & $6.19 \mathrm{E}-05$ & 0.8 & 0.00013 & 0.8 \\
\hline 1.025 & 8.822 & 82.32 & 6226 & $6.59 \mathrm{E}-05$ & 0.8 & 0.000133 & 0.8 \\
\hline 1 & 8.703 & 54.88 & 8705 & $7.24 \mathrm{E}-05$ & 0.8 & 0.000133 & 0.8 \\
\hline 0.975 & 8.588 & 36.44 & 12897 & $8.09 \mathrm{E}-05$ & 0.8 & 0.000132 & 0.8 \\
\hline 0.95 & 8.524 & 28.82 & 25812 & $8.88 \mathrm{E}-05$ & 0.8 & 0.000135 & 0.8 \\
\hline 0.925 & 8.428 & 21.16 & 167640 & 0.000101 & 0.8 & 0.000135 & 0.8 \\
\hline 0.9 & 8.363 & 16.68 & 145560 & 0.000113 & 0.8 & 0.000136 & 0.8 \\
\hline 0.875 & 8.301 & 13.71 & 512300 & 0.000127 & 0.8 & 0.000139 & 0.8 \\
\hline
\end{tabular}




\begin{tabular}{|c|c|c|c|c|c|c|c|}
\hline 0.85 & 8.228 & 11.13 & 100000 & 0.000144 & 0.8 & 0.00014 & 0.8 \\
\hline 0.825 & 8.356 & 11.56 & 382120 & 0.000168 & 0.80209 & 0.000126 & 0.82565 \\
\hline 0.8 & 8.339 & 10.94 & 1481100 & 0.000183 & 0.80764 & 0.00013 & 0.82929 \\
\hline \multicolumn{8}{|l|}{$10 \mathrm{~min}$} \\
\hline $\begin{array}{l}\text { Potential } \\
\text { (V) }\end{array}$ & $\mathrm{R}_{\mathrm{S}}(\Omega)$ & $\mathrm{R}_{\text {trapping }}(\Omega)$ & $\mathrm{R}_{\mathrm{ct}, \text { trap }}(\Omega)$ & $\begin{array}{l}\mathrm{CPE}_{\text {trap }}: \mathrm{T} \\
\left(\mathrm{F} \mathrm{cm}^{-2} \mathrm{~s}^{\mathrm{P}-1}\right)\end{array}$ & $\mathrm{CPE}_{\text {trap }}: \mathrm{P}(-)$ & $\begin{array}{l}\mathrm{CPE}_{\text {bulk }}: \mathrm{T} \\
\left(\mathrm{F} \mathrm{cm}^{-2} \mathrm{~s}^{\mathrm{P}-1}\right)\end{array}$ & $\mathrm{CPE}_{\text {bulk }}: \mathrm{P}(-)$ \\
\hline 1.7 & 11.05 & 8.534 & 16.06 & 0.000522 & 0.8 & 0.000166 & 0.8 \\
\hline 1.675 & 11.77 & 11.16 & 28.54 & 0.000483 & 0.8 & 0.000155 & 0.8 \\
\hline 1.65 & 12.54 & 16.87 & 55.51 & 0.000427 & 0.8 & 0.000153 & 0.8 \\
\hline 1.625 & 13.01 & 25.9 & 119.3 & 0.000384 & 0.8 & 0.000145 & 0.8 \\
\hline 1.6 & 13.33 & 40.2 & 234.1 & 0.000331 & 0.8 & 0.000139 & 0.8 \\
\hline 1.575 & 13.52 & 61.65 & 352.1 & 0.000296 & 0.8 & 0.000131 & 0.8 \\
\hline 1.55 & 13.58 & 91.09 & 427.4 & 0.000272 & 0.8 & 0.000122 & 0.8 \\
\hline 1.525 & 13.61 & 128.6 & 482.3 & 0.000261 & 0.8 & 0.000115 & 0.8 \\
\hline 1.5 & 13.74 & 167 & 536.7 & 0.000266 & 0.8 & 0.000111 & 0.8 \\
\hline 1.475 & 13.81 & 197.6 & 581.1 & 0.000272 & 0.8 & 0.000109 & 0.8 \\
\hline 1.45 & 13.88 & 219.8 & 616.8 & 0.000283 & 0.8 & 0.000107 & 0.8 \\
\hline 1.425 & 13.97 & 232.3 & 659.3 & 0.000289 & 0.8 & 0.000108 & 0.8 \\
\hline 1.4 & 14.04 & 239.6 & 697.3 & 0.000292 & 0.8 & 0.000108 & 0.8 \\
\hline 1.375 & 14.09 & 243.9 & 740.9 & 0.000293 & 0.8 & 0.000109 & 0.8 \\
\hline 1.35 & 14.11 & 245.8 & 804.3 & 0.000288 & 0.8 & 0.00011 & 0.8 \\
\hline 1.325 & 14.11 & 248.6 & 923.5 & 0.000287 & 0.8 & 0.000112 & 0.8 \\
\hline
\end{tabular}




\begin{tabular}{|c|c|c|c|c|c|c|c|}
\hline 1.3 & 14.13 & 251.5 & 1115 & 0.000285 & 0.8 & 0.000114 & 0.8 \\
\hline 1.275 & 14.19 & 252.9 & 1328 & 0.000276 & 0.8 & 0.000117 & 0.8 \\
\hline 1.25 & 14.2 & 253 & 1570 & 0.000265 & 0.8 & 0.000119 & 0.8 \\
\hline 1.225 & 14.22 & 253.5 & 1899 & 0.000253 & 0.8 & 0.000123 & 0.8 \\
\hline 1.2 & 14.24 & 252.7 & 2206 & 0.000243 & 0.8 & 0.000126 & 0.8 \\
\hline 1.175 & 14.24 & 253.4 & 2657 & 0.000232 & 0.8 & 0.000131 & 0.8 \\
\hline 1.15 & 14.26 & 256.2 & 3176 & 0.000223 & 0.8 & 0.000135 & 0.8 \\
\hline 1.125 & 14.25 & 256.9 & 3716 & 0.000214 & 0.8 & 0.00014 & 0.8 \\
\hline 1.1 & 14.24 & 258.3 & 4262 & 0.000206 & 0.8 & 0.000146 & 0.8 \\
\hline 1.075 & 14.24 & 260.7 & 4739 & 0.0002 & 0.8 & 0.000152 & 0.8 \\
\hline 1.05 & 14.23 & 265.1 & 5389 & 0.000194 & 0.8 & 0.000159 & 0.8 \\
\hline 1.025 & 14.24 & 265.4 & 6065 & 0.000189 & 0.8 & 0.000165 & 0.8 \\
\hline 1 & 14.2 & 256.2 & 6067 & 0.000184 & 0.8 & 0.000172 & 0.8 \\
\hline 0.975 & 14.18 & 260.5 & 6093 & 0.00018 & 0.8 & 0.00018 & 0.8 \\
\hline 0.95 & 14.2 & 263 & 6588 & 0.000177 & 0.8 & 0.00019 & 0.8 \\
\hline 0.925 & 14.17 & 259.2 & 6975 & 0.000175 & 0.8 & 0.000199 & 0.8 \\
\hline 0.9 & 14.14 & 257 & 6926 & 0.000173 & 0.8 & 0.000211 & 0.8 \\
\hline 0.875 & 14.13 & 259.3 & 6635 & 0.000171 & 0.8 & 0.000225 & 0.8 \\
\hline 0.85 & 14.09 & 249.8 & 6668 & 0.00017 & 0.8 & 0.000238 & 0.8 \\
\hline 0.825 & 14.05 & 246.1 & 6618 & 0.00017 & 0.8 & 0.000255 & 0.8 \\
\hline 0.8 & 13.98 & 237.7 & 6200 & 0.000168 & 0.8 & 0.000273 & 0.8 \\
\hline \multicolumn{8}{|l|}{$20 \mathrm{~min}$} \\
\hline
\end{tabular}




\begin{tabular}{|c|c|c|c|c|c|c|c|}
\hline $\begin{array}{l}\text { Potential } \\
\text { (V) }\end{array}$ & $\mathrm{R}_{\mathrm{S}}(\Omega)$ & $\mathrm{R}_{\text {trapping }}(\Omega)$ & $\mathrm{R}_{\mathrm{ct}, \text { trap }}(\Omega)$ & $\begin{array}{l}\mathrm{CPE}_{\text {trap }}: \mathrm{T} \\
\left(\mathrm{F} \mathrm{cm}^{-2} \mathrm{~s}^{\mathrm{P}-1}\right)\end{array}$ & $\mathrm{CPE}_{\text {trap }}: \mathrm{P}(-)$ & $\begin{array}{l}\mathrm{CPE}_{\text {bulk }}: \mathrm{T} \\
\left(\mathrm{F} \mathrm{cm}^{-2} \mathrm{~s}^{\mathrm{P}-1}\right)\end{array}$ & $\mathrm{CPE}_{\text {bulk }}: \mathrm{P}(-)$ \\
\hline 1.7 & 11.16 & 12.54 & 23.65 & 0.000573 & 0.8 & 0.000195 & 0.8 \\
\hline 1.675 & 11.46 & 16.79 & 34.16 & 0.000611 & 0.8 & 0.000199 & 0.8 \\
\hline 1.65 & 11.86 & 25.08 & 66.02 & 0.000582 & 0.8 & 0.000179 & 0.8 \\
\hline 1.625 & 12.26 & 35.34 & 133.4 & 0.000524 & 0.8 & 0.000168 & 0.8 \\
\hline 1.6 & 12.67 & 44.45 & 232.1 & 0.000457 & 0.8 & 0.000156 & 0.8 \\
\hline 1.575 & 12.88 & 61.35 & 313.2 & 0.000418 & 0.8 & 0.000149 & 0.8 \\
\hline 1.55 & 13.1 & 84.86 & 360.1 & 0.000387 & 0.8 & 0.000144 & 0.8 \\
\hline 1.525 & 13.22 & 114.1 & 381.7 & 0.000368 & 0.8 & 0.000139 & 0.8 \\
\hline 1.5 & 13.31 & 145.7 & 394.3 & 0.000368 & 0.8 & 0.000136 & 0.8 \\
\hline 1.475 & 13.41 & 175.3 & 410.9 & 0.000381 & 0.8 & 0.000134 & 0.8 \\
\hline 1.45 & 13.44 & 199.1 & 429.2 & 0.000396 & 0.8 & 0.000132 & 0.8 \\
\hline 1.425 & 13.49 & 215.2 & 462.2 & 0.0004 & 0.8 & 0.000133 & 0.8 \\
\hline 1.4 & 13.58 & 227.6 & 511.5 & 0.000392 & 0.8 & 0.000133 & 0.8 \\
\hline 1.375 & 13.64 & 235.8 & 569.2 & 0.000373 & 0.8 & 0.000133 & 0.8 \\
\hline 1.35 & 13.69 & 242 & 629.7 & 0.000349 & 0.8 & 0.000134 & 0.8 \\
\hline 1.325 & 13.74 & 250.1 & 743.9 & 0.000337 & 0.8 & 0.000137 & 0.8 \\
\hline 1.3 & 13.77 & 256.8 & 902 & 0.000324 & 0.8 & 0.000139 & 0.8 \\
\hline 1.275 & 13.79 & 260 & 1092 & 0.000307 & 0.8 & 0.000142 & 0.8 \\
\hline 1.25 & 13.8 & 267.1 & 1299 & 0.000293 & 0.8 & 0.000146 & 0.8 \\
\hline 1.225 & 13.8 & 275.9 & 1554 & 0.000279 & 0.8 & 0.00015 & 0.8 \\
\hline
\end{tabular}




\begin{tabular}{|c|c|c|c|c|c|c|c|}
\hline 1.2 & 13.77 & 283.4 & 1830 & 0.000263 & 0.8 & 0.000154 & 0.8 \\
\hline 1.175 & 13.76 & 295.4 & 2165 & 0.000248 & 0.8 & 0.000159 & 0.8 \\
\hline 1.15 & 13.73 & 307.3 & 2603 & 0.000233 & 0.8 & 0.000164 & 0.8 \\
\hline 1.125 & 13.7 & 320.1 & 2998 & 0.000217 & 0.8 & 0.00017 & 0.8 \\
\hline 1.1 & 13.68 & 334.6 & 3462 & 0.000202 & 0.8 & 0.000176 & 0.8 \\
\hline 1.075 & 13.64 & 347.2 & 3912 & 0.00019 & 0.8 & 0.000183 & 0.8 \\
\hline 1.05 & 13.62 & 364.8 & 4413 & 0.000179 & 0.8 & 0.00019 & 0.8 \\
\hline 1.025 & 13.58 & 382.2 & 4919 & 0.00017 & 0.8 & 0.000197 & 0.8 \\
\hline 1 & 13.54 & 389.6 & 5241 & 0.00016 & 0.8 & 0.000204 & 0.8 \\
\hline 0.975 & 13.52 & 401.5 & 5585 & 0.000155 & 0.8 & 0.000213 & 0.8 \\
\hline 0.95 & 13.49 & 415.9 & 5746 & 0.000149 & 0.8 & 0.000222 & 0.8 \\
\hline 0.925 & 13.46 & 438 & 6023 & 0.000145 & 0.8 & 0.000233 & 0.8 \\
\hline 0.9 & 13.44 & 439.3 & 5935 & 0.000141 & 0.8 & 0.000244 & 0.8 \\
\hline 0.875 & 13.41 & 465.2 & 6028 & 0.000139 & 0.8 & 0.000257 & 0.8 \\
\hline 0.85 & 13.37 & 468.9 & 5720 & 0.000134 & 0.8 & 0.000271 & 0.8 \\
\hline 0.825 & 13.35 & 502.4 & 5730 & 0.000135 & 0.8 & 0.000287 & 0.8 \\
\hline 0.8 & 13.33 & 515.6 & 5259 & 0.000135 & 0.8 & 0.000304 & 0.8 \\
\hline
\end{tabular}

